\title{
AUTONOMOUS MULTI-AGENTS ARCHITECTURE FOR CONTROL OF MANUFACTURING SYSTEMS
}

\author{
B. Bouzouïa ${ }^{1}$, Y. Hafri ${ }^{2}$, T. Boudellal ${ }^{2}$ \\ 1: Centre de Développement des Technologies Avancées. 128 Mohamed Gacem. \\ El-Madania, Algiers (Algeria). Email: brahim.bouzouia@cdta.dz \\ 2: University of Sciences and Technologies of Houari Boumediene. BP 32 El Alia \\ Bab Ezzouar, Algiers (Algeria).
}

This paper focuses on the discussions of innovative Multi-Agent approach to advanced real time control for flexible manufacturing systems. The architecture is based on the paradigm of Distributed Intelligence and MultiAgent systems. This Multi-Agent prototype system involves the following functions: Scheduling, dispatching, monitoring and error handling. A new negotiation protocol for manufacturing system is also presented in this paper. The purpose of this protocol is to dynamically assign operations to the resources of the Manufacturing System in order to accomplish the proposed tasks. This protocol is able to deal with exceptions.

\section{INTRODUCTION}

Global competitiveness has been imposing deep changes to all components of manufacturing systems, including the real time scheduling. From an emphasis on the scheduling optimality in the past, the focus has later moved to scheduling flexibility[1].

The function of real time cell control system can vary depending on the size of a cell, its type and the degree of decision-making capabilities given to a cell. The major functions of a cell control system include the need to schedule and monitor cell resources, and the ability to react to abnormal condition or exceptions [2] [3].

The major approaches of scheduling are represented by the traditional operational research techniques as combinatorial procedures, heuristic approaches and constraint analysis [4][5]. Nevertheless, this approaches are NP-complet and has shown many limitations. Such systems soon became too heavy to be implemented as a centralised control program in only one computer. Fortunately, distributed computing has already given some answers to the problem of how to implement efficiently communities of interactive systems. This emerging area is a Distributed Artificial Intelligence (DAI)[5][6][7]. The framework of DAI, particularly Multi-Agent System (MAS), seams more suitable for the dynamic control of manufacturing 
systems. The Multi-Agent System paradigm represents one of the most promising approach to build complex and flexible advanced intelligent systems. The application of MAS approach in dynamic scheduling and control is based on the idea that the scheduling and control agility can be extremely improved once there are based on the following key points: i) distributed and autonomous systems instead of centralised and non-autonomous solutions; ii) negotiation-based decision making instead of the totally pre-planned processes; iii) uses of different problem-solvers in the same environment instead of only one fixed problem solver; and iv) concurrent execution instead of sequential processing. Several works on flexible manufacturing system control followed the paradigm of DAI. Here we will refer only two of these systems: ICOSS and MASSYVE. ICOSS [8] (Intelligent Cell Objects/Intelligent Supporting Shell) architecture consists of two layers: the inner layer called ICO contains cell control knowledge that perform generic cell control functions such as scheduling, dispatching, monitoring; the outer layer called ISS contains cell databases that represents the specific cell environment. The two layer approach allows more efficiency in developing and implementing intelligent cell control by considering generic cell control knowledge and cell-specified databases separately. MASSYVE [9] (Multi-Agent Agile manufacturing Scheduling SYstems for Virtual Enterprises) aims to investigate the use of multi-agent systems in agile scheduling, towards the operation in a virtual enterprise environment. These activities use the HOLOS framework as base line for advanced scheduling. The information integration approach supporting a multi-agent system in MASSYVE is based on the PEER information management framework.

This paper presents a new Architecture and Negotiation Protocol (JTransactions Machines) for an intelligent cell control system. We consider that requests involve due dates (deadlines) for the tasks to be carried out and some applications of this framework to Distributed Robotic Systems. Section II presents a new architecture for real time scheduling of Manufacturing Systems. Section III and IV illustrate how the negotiation/renegotiation protocol is established. Finally, conclusions are presented in section $\mathrm{V}$.

\section{A NEW ARCHITECTURE FOR DYNAMIC SCHEDULING AND CONTROL OF FMS}

An automated manufacturing cell typically consists of numerical control machines, industrial robots, storage devices, automatic inspection devices, tools and fixtures and control computers. The machine tools in the cell are physically interconnected by automated material handling devices such as conveyors, AGVs and robots. Communication networks provide the information links within the cell.

Fig.1 illustrates the Architecture proposed in this paper. This architecture includes, essentially, two parts : one concerning resources and another concerning agents. The number of resources does not vary, except when resources are introduced or removed from the Manufacturing System.

The system architecture contains the following agents : 
Initiator Agent : interfaces with the user receiving orders of new tasks for the Manufacturing System. This agent is responsible for launching Task Announcement Message whenever a new task is ordered (starts the execution of scheduling process). It is also maintain global view on the activity of the resources(Mirrors Agents).

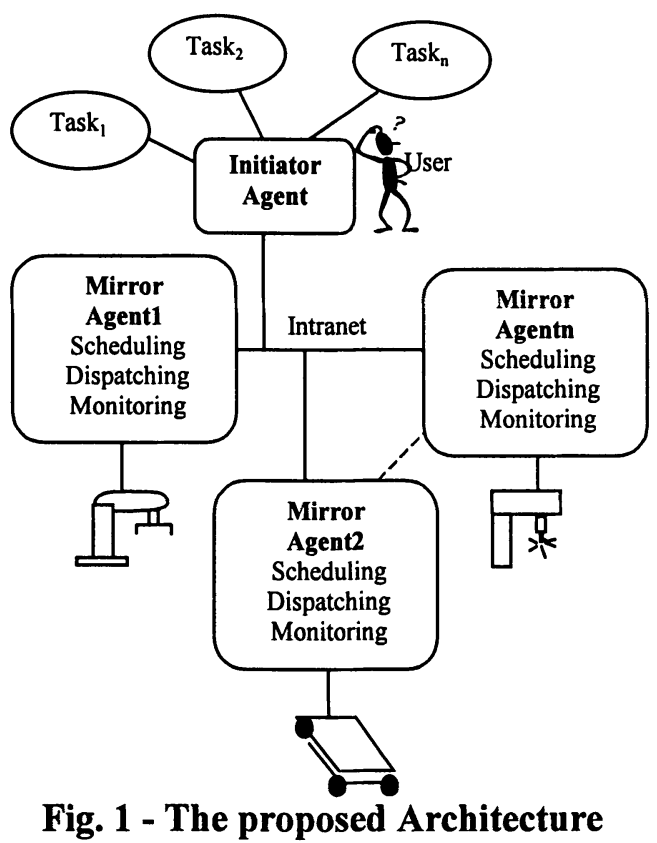

Mirror Agent (MA): an autonomous agent called Mirror Agent represents every resource in the cell. $\mathrm{MA}_{\mathrm{i}}$ represents the current situation of resource $i$ (its status and activity). The activity is a sequence of operation to be carried out which is represented into an agenda. This Agent is, also, responsible to perform the four control functions, which are: scheduling, dispatching, monitoring and error handling.

For a better output, two production targets direct the control system:

- The reduction of the number of late tasks: The initiator agent will start by seeking the tasks in the following order: tasks for rescheduling, urgent tasks and finally shortest tasks.

- The manufacture of the products to rather: The mirror agent to contract an operation is that which offers the completion date nearest to the current date.

\section{NEGOTIATION PROTOCOL (JTransactions Machines, JT.M)}

Let us suppose the example of fig. 2, which consists to produce $\mathbf{Q}$ parts $\mathbf{X Y}$. The part must be polish on front and drill on sides in a specific deadline DL. 
Feed_drilling_machine(xy) AND opr 1 - drill(xy) $\rightarrow$ Xy part.

Feed_turning_machine(Xy) AND opr 2 - polish(Xy) $\rightarrow$ XY part AND opr 3 - transport $(X Y)$

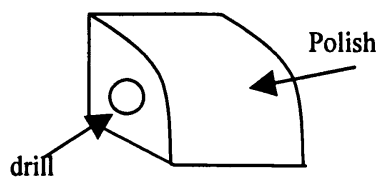

Fig. 2 - List of operations for the XY part

The JT.M protocol is based on the Contract-Net protocol proposed in [10]. However, this protocol improves the basic behaviour of Contract-Net by the distribution of control functions over all the agents of the system. The fig. 3 and 4 illustrate the Negotiation Protocol for contracting resources for execution of task. When a new request for task execution appears, the user specifies the name of the task to be carried out as well as its deadline. In order to guarantee the deadline, the negotiation between the Mirror Agents is performed in backward chaining. The negotiation can be summarised in the following steps:

Note: All messages supported by the control system contains the fields: \{SEN, $A D R\}$ where $S E N$ is a sender of the message and ADR is the addressee.

- Step one:

The Initiator Agent extracts a last operation of the task and constructs, using knowledge about the material configuration, the list of possible resources for the operation (fig. 3). The Mirror Agents corresponding to this operation receive a message with the following format :

$\{C D, T D, Q, D L, L O C\}$

Where : CD is customer identifier, TD is a task descriptor, $\mathrm{Q}$ is a number of operation sequences(e.g. 5 objects XY for the example of fig. 2), DL is deadline for a task delivery(date and time) and LOC is the list of operations including their constraints.

This message will be referred as the "Task Announcement Message ' (T.A.M).

- Step two:

The Mirror Agent, that had received Announcement proceed as follows:

It extracts all the operations which is concerned by from the task (operations are considered from last, last but one,...). After that, the Mirror Agent tests the possibility to carry out at least the last operation before a deadline DL. If so, it sends 'Request Messages' (R.M) on the (N-k), where k is a number of operations extracted by Mirror Agent, operations corresponding to the sub-hierarchy of operations (fig. 3). The propagation of requests is reiterated until there is no operation to negotiate. The Request Message has the following format :

$\left\{C D, T D, Q_{i}\right.$, DL, LOC, NLO, SD, TDRR, CO $\}$

Where: $Q_{i}$ is a maximum amount of operations to guarantee the deadline by sender $\left(\mathrm{MA}_{\mathrm{i}}\right)$ of request, NLO is the list of remaining operations, SD is the time 
where the $Q_{i}$ operations could be started, TDRR is a temporal delay required by the SEN to ADR on propagation request phase and $O C$ are the constraints related to the operative duration preceding current request.

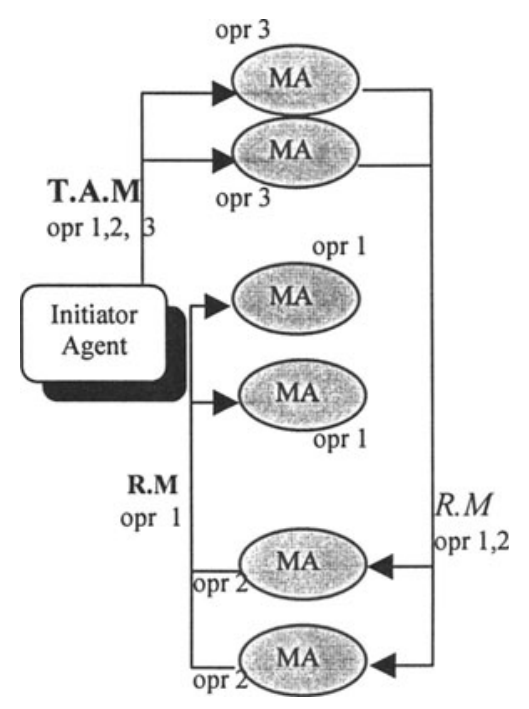

Fig. 3 - Task Announcement and

Request Propagation

\section{- Step three:}

The Mirror Agents which receive request for elementary operations are the first whose broach intra-level negotiation (this agents controls a same resources). Instead that, they proceed as follows:

Each $M A_{i}$ sends to its contacts the information's about its capacity to make $Q_{i}$ operations among the amount $\mathrm{Q}\left(\mathrm{Q}_{\mathrm{i}}<=\mathrm{Q}\right.$ and due date $\mathrm{DL}_{\mathrm{i}}$ for these $\mathrm{Q}_{\mathrm{i}}$ operations $\mathrm{DL}_{\mathrm{i}}<=\mathrm{DL}$ ). Candidates start a same algorithm for the selection of the best agents. Therefore, each one obtains an identical results and known if it participates or not at the production of XY parts(fig. 4).

Note: - The criterions of selection favours Agents that offers an earliest date of manufacturing and consider the possibility to split the operations by several resources $\left(\Sigma \mathbf{Q}_{i}=\mathbf{Q}\right)$.

- An agent can negotiate with null values if it did not receive invitations to tender concerning the task in progress.

The 'Negotiation Message'(N.M) has the following format :

$\left\{C D, Q_{\max (i)}, D_{\max (i)}\right\}$

Where: $\mathrm{Q}_{\max (\mathrm{i})}$ and $\mathrm{DL}_{\max (\mathrm{i})}$ are the maximum number of operations and their deadline which Mirror ${ }_{i}$ of the last level can assure. 


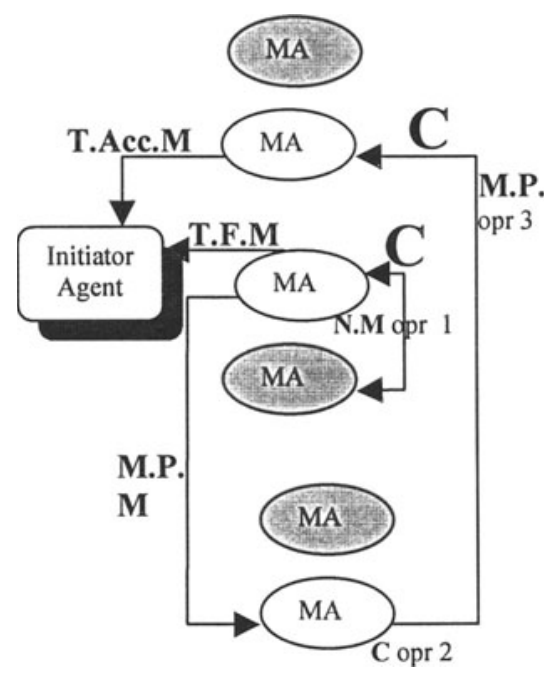

Fig. 4 - Negotiation, Contract and Proposition Feedback

\section{- Step four:}

After that, the Mirror Agents selected establishes their contracts $(C)$ and send 'Mirror Proposition Messages' (M.P.M) to Mirrors of the up-hierarchy (fig. 4). The Mirror Agent, which is concerned by the last operation of all the XY parts, with the reception of the proposition message detects the end of the scheduling process and sends an 'Task Acceptance Message' (T.Acc.M) to the Initiator Agent (fig. 4).

The Mirror Proposition Message has the following format:

$\left\{C D, T D, Q_{i}, D D, D_{i}, T D R B, C O\right\}$

Where: TDRB is a temporal delay required by the SEN to ADR on proposition feedback phase.

On the other hand, if Mirror Agents are not able to guarantee the deadline $\left(\sum \mathrm{Q}_{\mathrm{i}}<\mathrm{Q}\right)$ the negotiation is finished without contract and a 'Task Failure Message'(T.F.M) is sent to the Initiator Agent by Mirror which offers the best criterions in intra-level negotiation phase(fig. 4).

\section{Dispatching, Monitoring and Error handling}

First, the module extracts a contract (operation) from its agenda and sends its preconditions to the inference engine that verifies them. The precondition operation calls the sensory equipment of the resource in order to obtain data concerning the presence of the part, its identity and to compare them with the initial status of reference. In the favourable case (no exceptions are detected after the inference), the operation is launched and a starting order is sent to the resource controller.

When the operation is finished, the resource controller sends a signal specifying the operation characteristic to the inference engine. After that, the postcondition operation is launched. This operation, contrary to the first, checks the status of the 
operation carried out and compares them with the final status of reference. If no exceptions appear, the dispatching module executes the next operation (fig. 5).

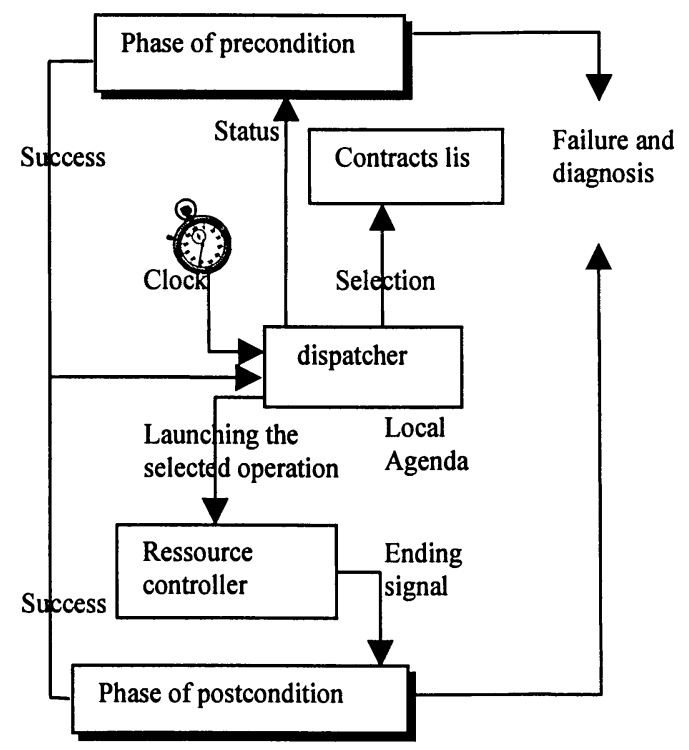

Fig. 5 - Operation execution mechanism

\section{The inference engine:}

After an anomaly detection, the diagnosis module (figure.6) proceeds by:

- $\quad$ Formatting facts and loading them in the facts base,

- $\quad$ Launching the inference.

It sends thereafter, the results of the inference to the concerned process (communication, rescheduling, etc).

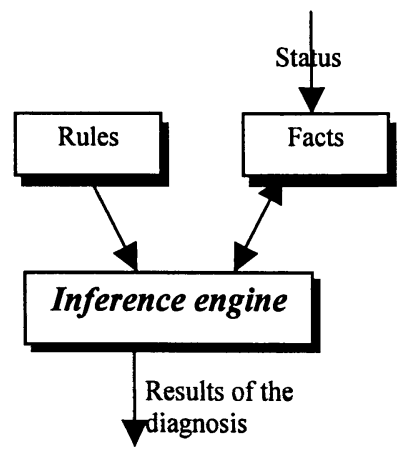

Fig. 6 - Diagnosis module 


\section{EXCEPTIONS AND RENEGOTIATION}

Once a task has been accepted it will be accomplished in the specified deadline if no exceptions appear. The Mirror Agents sends starting orders to there appropriate resources and wait for ending signal or abnormal events. However, exceptions (e.g. machine in failure or operation in fault) may happen and manufacturing system control has to deal with this kind of problems.

The Mirror Agents support Monitoring function that recognises and analyses exceptional conditions or errors of their resource, and provides a possible corrective actions to these problems (renegotiation, operator call,...). When Mirror Agent executes an operation, its sensory equipment informs the Monitoring about the status of operation, resource or parts. This information is sent to the Error handling to diagnose the errors by the use of production rules based on predicate logic formalism.

For comprehensive reasons, we suppose that the product $\mathrm{XY}$ is assigned to a productive way between Mirror Agents MA1, MA3 and MA6. The Mirror Agent related with the exception (fig. 7, MA3) establishes a list for all contracts affected by the failure. After that, it will send an 'Contract Cancellations' messages to Agents appears in the list, as well as, an 'Operation Failure' message to Initiator Agent enclosing the amount of non accomplished operations. Finally, it destroys the list.

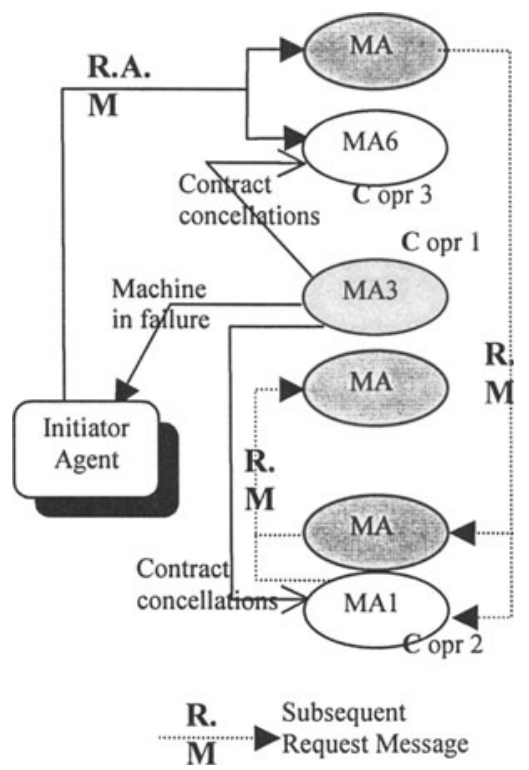

Fig.7 - Renegotiation protocol

The Operation Failure Messages has the following format: $\{$ CD, TD, ANO $\}$. Where: ANO is the amount of non accomplished operations. 
The Contract Cancellations Messages has the following format: \{CD, TD, LCAF\}.

Where: LCAF is a list of contracts affected by the failure. The Initiator Agent will send a priority 'Task Announcement Message' for the rescheduled client.

If Mirrors Agents do not allow the accomplishment of the deadline then a 'Task Failure Message' is sent from the Mirror Agent(see the intra-level negotiation phase) to the Initiator, otherwise, a message is sent to inform that task has been accepted.

\section{IMPLEMENTATION ISSUES}

\section{Software Architecture}

The system is implemented in Object Oriented Programming with JAVA2 (Sun Micro System) that allows multi-threading and supports agents executing on different platforms (Microsoft Windows 9X, UNIX, OS2). The Agents of the system use TCP/IP protocol for communication and apply a recursive algorithm for sending/receiving Message Object. Finally, the error handling is implemented with JESS5.0a6 (Java Expert Shell System of Sandia National Laboratories Livermore, CA).

\section{Material Architecture}

The flexible cell on which we have applied our control system is formed by a conveyor, a CNC turn machine and a handling GT6 robot.

The resources are controlled by three Pentium II 300Mhz microcomputers plus one for the operator, interconnected by a local network having a star topology (fig.8).

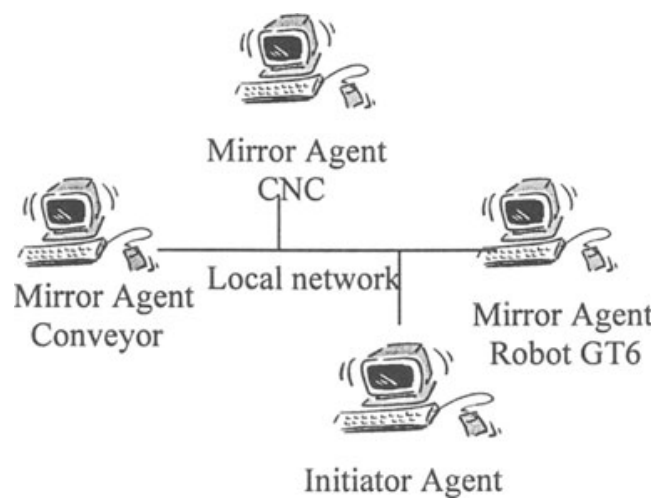

Fig.8 Material Architecture

\section{CONCLUSION}

This paper addresses a cell dynamic scheduling and control architecture that applies Multi-Agents approach to the problem of flexible manufacturing systems (FMS). It 
also presented a new Negotiation Protocol (JT.M) suitable for the dynamic scheduling of manufacturing tasks. This Negotiation Protocol is able to deal with exceptions, since a renegotiation phase of the protocol can be activated. The main advantages of this architecture are : agent autonomy, decentralised decision-making capability, flexibility in more changing environment, sophisticate communication system, increased fault-tolerance, real time behaviour, agents are totally co-operative and inherent reconfiguration. Control functions (scheduling, dispatching, monitoring, error handling) are totally distributed over the entire agents in the system. There are also other functions, such as: cell initialisation, communication, user interface and tools that support visualisation of agent performance.

\section{References}

[1] D Trenteseaux. Conception d'un Système de Pilotage Distribué, Supervisé et Multicritère pour les Systèmes Automatisés de Production, Thèse de doctorat à l'Institut National Polytechnique de Grenoble, France, 1996.

[2] D. Ouelhadj, C. Hanachi, B. Bouzouïa, A. Moualek, A. Farhi. A multi-contract net protocol for dynamic scheduling in flexible manufacturing systems. IEEE, International Conference on Robotics \& Automation Detroit, Michigan, USA, 1999.

[3] D.Ouelhadj, C. Hanachi, B. Bouzouïa. Multi-Agent system for dynamic scheduling and control in manufacturing cells. ICRA'98, IEEE International Conference on Robotics and Automation, Louvain (Belgium), 16, 20 mai, 1998.

[4] S. Balasubramania, D.H Norrie A Multi-Agent Intelligent Design System Integrating Manufacturing And Shop Floor Control, University of Calagray, Canada, AB, T2N IN4, 1997.

[5] J. Ferber and M.Ghallab. Problématique des univers multi-agents intelligents. Journées nationales PRC-GRECO. Intelligence Artificielle, page 295-320, Toulouse, France, mars 1999.

[6] C. Ramos. Architecture and Negotiation protocol for the dynamic scheduling of manufacturing systems. Proceedings of IEEE International Conference on Robotics and Automation, Vol.4, Page 8-13, 1994.

[7] J. Ferber. Les Systèmes Multi-Agents: Vers une Intelligence Collective. $\mathrm{n}^{\circ}$ ref. 5548.4 1174, iia 1995.

[8] K.H Lee and S. Sen. ICOSS : A Two-Layer. Object Based Intelligent Cell Control Architecture Computer Integrated Manufacturing Systems, 7(2) 100_112 Department of Industrial Engineering, Northern Illinois University, DeKalb, IL 60115-2854, USA, 1994.

[9] R. Rabelo, L.M. Camarinha-Matos, H. Afsarmanesh. Multi-agent-based agile scheduling, Journal of Robotics and Autonomous Systems (Elsevier), Vol. 27, N. 1-2, Abril 1999, ISSN 0921-8890, pp. 15-28.

[10] G.S Reid. The Contract-Net protocol: High level communication and control on a distributed problem solver. IEEE Transactions on Systems. Man \& Cybernetics. 